\title{
A Betalain-rich Dietary Supplement, But Not PETN, Increases Vasodilation and Nitric Oxide: A Comparative, Single-dose, Randomized, Placebo-controlled, Blinded, Crossover Pilot Study
}

\author{
B. V. Nemzer ${ }^{1,2}$, Z. Pietrzkowski ${ }^{3}$, J. M. Hunter ${ }^{2}$, J. L. Robinson ${ }^{4}$ \& B. Fink ${ }^{5}$ \\ ${ }^{1}$ VDF FutureCeuticals, Inc., Momence, IL, USA \\ ${ }^{2}$ University of Illinois at Urbana-Champaign, Momence, IL, USA \\ ${ }^{3}$ VDF FutureCeuticals, Inc., Irvine, CA, USA \\ ${ }^{4}$ Department of Psychology, Auburn University, AL, USA \\ ${ }^{5}$ Noxygen Science Transfer \& Diagnostics GmbH, Elzach, Germany \\ Correspondence: B. V. Nemzer, VDF FutureCeuticals, Inc., Momence, IL, USA. Tel: 1-815-507-1427. E-mail: \\ bnemzer@futureceuticals.com
}

Received: November 7, 2020

Accepted: December 17, 2020 Online Published: December 22, 2020

doi:10.5539/jfr.v10n1p26

URL: https://doi.org/10.5539/jfr.v10n1p26

\begin{abstract}
Nutraceutical supplements have demonstrated promise as agents for improving athletic performance and for positively affecting cardiovascular health and vigor through modulation of endothelial function at the cellular level. High-nitrate products, such as red beet juices and powders, have been observed to improve athletic performance potentially through increased nitric oxide (NO) concentrations in the blood. Similarly, a patented low nitrate, low sugar betalain-rich supplement has also been reported to significantly improve athletic performance. To the best of our knowledge, no acute clinical studies have been conducted that have demonstrated the comparative efficacies of high-nitrate or betalain-rich, low nitrate materials on measures of endothelial function in real time. In this acute single-dose, double-blinded, randomized, placebo-controlled, crossover study, we examined the effects of the betalain-rich low nitrate dietary supplement, (BRS, 50mg), in comparison to pentaerythritol tetranitrate (PETN, 40mg), a pharmaceutical drug that is a potent source of organic nitrate, and a placebo, on various measures of endothelial function for up to 4-hours post-ingestion. More specifically, in order to gauge post-treatment changes in endothelial function we measured flow-mediated dilation (FMD), nitrite $\left(\mathrm{NO}_{2}\right)$ /nitrate $\left(\mathrm{NO}_{3}\right)$ content, circulating nitrosyl-hemoglobin $(\mathrm{NOHb})$ concentration, and cellular metabolic activity (CMA) measured as generation of reactive oxygen species, a side reaction of oxidative-reductive cellular metabolism. Ten participants completed all arms of the study. Results suggest that within 2 hours, BRS, but not PETN or placebo, resulted in significantly elevated levels of $\mathrm{NOHb}$ (a measure of bioavailable $\left.\mathrm{NO}^{\circ}\right)(p=0.017)$ and increased vasodilation as measured by FMD, $(p=0.025)$. As expected, due to its high nitrate content, $\mathrm{NO}_{2} / \mathrm{NO}_{3}$ levels were increased by PETN within 2-hours $(p=0.048)$, but not by BRS or placebo. Finally, under these experimental conditions, PETN and BRS produced no significant changes for mitochondrial, NADPH-oxidase dependent or cellular CMA. These data provide preliminary support for single-dose effectiveness of BRS, but not PETN, on levels of bioavailable $\mathrm{NO}^{\bullet}$ and FMD, both important measures of endothelial function. Additionally, these data suggest potentially different mechanisms of action related to low nitrate BRS and organic nitrate PETN.
\end{abstract}

Keywords: vasodilation, cellular metabolic activity, circulating $\mathrm{NOHb}, \mathrm{NO}_{2} / \mathrm{NO}_{3}, \mathrm{PETN}, \mathrm{FMD}$, herbal supplement, betalains, BRS, AltRed ${ }^{\mathrm{TM}}$, nutraceutical, nitrates, beets

\section{Introduction}

Recently, there has been significant interest in natural products that may promote improvements in athletic performance or, in general, support cardiovascular health. Since the mid-1800s, nitroglycerine has been used therapeutically as a vasodilator for treatment of "angina pectoris". This supports the concept that dysfunction of the endothelium results in impaired $\mathrm{NO}^{\bullet}$ generation. Healthy function of the vascular endothelium is maintained by an equilibrium based upon the bioavailability of $\mathrm{NO}^{\bullet}$, and relative levels of reactive oxygen species (ROS) as 
superoxide $\left(\mathrm{O}_{2} \cdot\right.$ ) (Landmesser et al., 2002), hydrogen peroxide $\left(\mathrm{H}_{2} \mathrm{O}_{2}\right)$ (Lacy, O'Connor, \& Schmid-Schönbein, 1998), and peroxynitrite (ONOO-) (Beckman \& Koppenol, 1996). Within the vascular endothelium, $\mathrm{NO}^{\bullet}$ induces vasodilatation that initiates a host of downstream consequences. These include increased oxygen delivery, reduction in platelet aggregation and adhesion, prevention of smooth muscle proliferation, inhibition of adhesion of leukocytes and expression of pro-inflammatory cytokine genes. It also helps to counteract lipid oxidation. A shift in the balance between $\mathrm{NO}^{\bullet}$ and ROS that favors $\mathrm{NO}^{\bullet}$ deficiency causes increases in ROS formation and leads to endothelial dysfunction. Virtually all cells in the vessel wall (i.e., endothelial, smooth muscle and adventitial cells) and in various enzymatic systems produce ROS albeit in different amounts and in response to diverse stimuli (Doehner \& Landmesser, 2011; Doughan, Harrison, \& Dikalov, 2008). These varying amounts of ROS can subsequently act in an autocrine or paracrine fashion to modulate cellular function (Griendling, Sorescu, \& Ushio-Fukai, 2000). Interestingly, endothelial nitric oxide synthase (eNOS) is the source of $\mathrm{NO}^{\bullet}$ in the vascular endothelium, and the uncoupling of eNOS defines endothelial dysfunction (Laude et al., 2005). Plasma nitrite has been shown to correlate with changes in eNOS activity by unchanged generation of ROS, thus providing one avenue for indirect assessment of endothelial function (Kleinbongard et al., 2003; Kleinbongard et al., 2006).

Over the past two decades, researchers have been investigating the role and involvement of endogenous and exogenous sources of $\mathrm{NO}^{\bullet}$ in the human body in order to find a way to overcome the phenomenon of endothelial dysfunction and to potentially enhance athletic performance. An important aspect of these investigations required the development of improved technologies for the direct measurement of bioavailable $\mathrm{NO}^{\bullet}$ levels. We previously developed and reported one such method for direct measurement of bioavailable NO ${ }^{\bullet}$ (Dikalov \& Fink, 2005; Fink, Dikalov, \& Fink, 2006; Nemzer et al., 2014) and have employed it to generate the data presented herein. Several other measures are indicative of vascular endothelial function including flow mediated dilation (FMD, a predictor of endothelial function; Ras, Streppel, Draijer, \& Zock, 2013; Flammer et al., 2012), superoxide generation, cellular ROS, and lipid peroxidation. The assessment of levels of ROS and lipid peroxidation is important in order to better understand endothelial dysfunction (Bibli et al., 2020; McDonald et al., 2018; Ramana, Srivastava, \& Singhal, 2014). Contributing to the novelty of this study, the use of such an array of measures in an acute administration design allows for a comprehensive assessment of endothelial health.

The use of organic drugs such as nitrate nitroglycerine (GTN) or isosorbide dinitrate (ISDN) for the treatment of angina pectoris have been employed as an option to support impaired endothelial function. Unfortunately, such compounds undergo oxidative bioconversion, especially during nonintermittent treatment, thereby causing the development of nitrate tolerance, which can be circumvented by dietary supplementation of vitamin C (Bassenge, Fink, Skatchkov \& Fink, 1998; Dikalov, Fink, Skatchkov \& Bassenge, 1999). Organic nitrates such as pentaerythritol tetranitrate (PETN) undergo reductive bioconversion and slowly release NO. Vascular tolerance can be avoided by nonintermittent but low dose (40 mg BID) treatment with these nitrates; however, they have been reported to elicit moderate nitrate tolerance at high doses (80 mg BID) (Fink \& Bassenge, 1997; Fink \& Bassenge, 2002).

More recently, natural, plant-based materials that contain high levels of nitrates (e.g., red beetroot powders/juices) or bioactive polyphenols or other phytochemicals have been of particular interest (Hurtado-Barroso et al., 2018; Mendonça et al., 2018), given preliminary evidence that they support healthy endothelial function and may enhance athletic performance (Montenegro, Kwong, Minow, Davis, Lozada \& Casazza, 2017), as potential ways to bridge this clinical gap. For example, betalains, a relatively new class of plant-derived antioxidants found in red beets, have been shown to alter lipid peroxidation (Kanner, Harel \& Granit, 2001) and may have downstream effects on vascular endothelial function. Thus, it is reasonable to consider whether nutraceuticals with high concentrations of betalains may aid in supporting athletic performance, and may support improved $\mathrm{O}_{2}$ utilization, healthy metabolism, and healthy cardiovascular systems. One such material is the betalain-rich supplement (BRS) that is the focus of the current study. This material is characterized by a high concentration of betalains (please see Table 2) and, unlike typical beetroot materials, it contains extremely low (insignificant) concentrations of inorganic nitrates and sugars, and other ingredients such as amino acids, and vitamins. Earlier clinical studies on BRS reported significant improvements in athletic performance in runners (Hoorebeke, Trias, Davis, Lozada \& Casazza, 2016) and cyclists (Montenegro et al., 2017). Additionally, preliminary clinical studies on BRS have exhibited increases in NO, hematocrit, and erythropoietin (EPO) (in house, unpublished data). Despite its promise as a nutraceutical with beneficial athletic performance and cardiovascular benefits, BRS has not been clinically tested to determine its specific effects on endothelial function or the potential pathophysiological mechanisms subserving such effects.

Here, we conducted a pilot clinical study to measure the effects of BRS, a low nitrate, betalains-rich dietary 
supplement, in comparison to PETN, a high organic nitrate pharmaceutical, and a placebo in connection to NO ${ }^{\bullet}$ formation and bioavailability related to vascular endothelial function. More specifically, we performed a double-blind, placebo-controlled, randomized cross-over clinical study observing the single-dose, time-dependent effects of BRS, PETN, and placebo on the following primary outcomes: a.) flow-mediated vasodilation (FMD); and, b.) circulating $\mathrm{NOHb}$ levels in blood (NOHb); and, c.) $\mathrm{NO}_{2} / \mathrm{NO}_{3}$ concentration in plasma using the Griess method; and, d.) cellular metabolic activity (CMA) in order to verify levels of i.) superoxide generation (NADPH-dependent) and ii.) cellular ROS, e.) levels of lipid peroxidation We hypothesized that BRS would increase FMD and $\mathrm{NOHb}$ but, due to its low nitrate content, it would not significantly increase $\mathrm{NO}_{2} / \mathrm{NO}_{3}$. We also hypothesized that both BRS and PETN would reduce lipid peroxide concentrations and decrease ROS generation as measured by CMA. We further hypothesized that, due to previous reports that high nitrate products showed enhanced athletic performance, PETN would likely increase FMD and $\mathrm{NOHb}$ and, again due to its high nitrate content, it would also increase $\mathrm{NO}_{2} / \mathrm{NO}_{3}$. Furthermore, we predicted that placebo would have no effect on any of these measures.

\section{Materials and Methods}

\subsection{Participants}

Fourteen participants were screened for participation in the study. Based on this study protocol, the inclusion criteria to recruit healthy volunteers were 30-50 years old, BMI 20-24, 5 males and 5 females, with healthy endothelial function (FMD $>12 \%$, HbNO level $>100 \mathrm{nM})$ and healthy CMI $(<220 \mathrm{nM} / \mathrm{sec})$. The exclusion criteria eliminated individuals with diagnosed Type 1 or Type 2 diabetes, other acute or chronic disorders (gastrointestinal, pulmonary, renal, cardiac, neurological or psychiatric disorders), individuals with known allergies to any food or ingredient and participants that were active smokers, using weight-reducing preparations/appetite suppressants, $\beta$-blockers, ACE inhibitors or who had participated in a clinical study within the last 90 days before the beginning of this study. According to these criteria four participants were excluded from this study: 2 with the CMA values over $220 \mathrm{nM} / \mathrm{sec}$, one with FMD < 12\% (6\%) and one with NOHb < $100 \mathrm{nM}(58 \mathrm{nM})$. Baseline measures of FMD, NOHb, and CMA levels were determined for all participants. Ten volunteers participated in the study $(5$ males $/ 5$ females, age $(M \pm S D)=35.90 \pm 9.71)$. All participants were in generally good health as confirmed by physical examinations and clinical routine laboratory tests. Detailed demographic information can be found in Table 1. Participants provided written, informed consent.

\subsection{Study Design}

This study was conducted using a randomized, single-dose, placebo-controlled, double-blind, crossover design. Specifically, participants were randomized based on an ordering of 4 possible conditions, of which 3 conditions were of interest for the current manuscript. The conditions were: placebo, PETN (40mg), BRS (50 mg), and another commercially available herbal supplement that is not presented within the current manuscript. Data related to this fourth condition were omitted because said material a.) had a different method of delivery (beverage vs capsules); b.) required a significantly different dosage compared to the other arms which c.) consequently made it impossible to accomplish a double blind protocol; and, d.) was included only as a convenience in order to obtain some early, exploratory information for future study designs. Fasting participants were given a single dose of the liquid herbal supplement, or PETN, BRS or placebo capsules with a glass of water, on four different days, separated by a wash-out period of 3 days. After the 12-hour fasting period, venous and capillary blood was collected before supplementation, and subsequently 1-, 2-, 3- and 4-hours after ingestion of the capsule to measure endothelial flow-mediated vasodilation (FMD), circulating $\mathrm{NOHb}, \mathrm{NO}_{2} / \mathrm{NO}_{3}$ content, NADPH oxidase dependent CMA, cellular CMA (representing the leakage of electrons of all cellular reductive-oxidative metabolic processes), blood cells lipid peroxide concentration. 
Table 1. Clinical laboratory parameters of test subjects

\begin{tabular}{lllll}
\hline & Parameters & Mean & SD & Unit \\
\hline Antropometry & Age & 35.90 & 9.71 & years \\
& BMI & 24.20 & 3.06 & \\
& SBP & 109.90 & 10.03 & $\mathrm{mmHg}$ \\
Cardiovascular health & dBP & 67.50 & 6.00 & $\mathrm{mmHg}$ \\
& CMA Total & 241.20 & 9.10 & $\mathrm{nM} / \mathrm{sec}$ \\
& NOHb & 143.89 & 29.09 & $\mathrm{nM}$ \\
Glucose profile & FMD & 8.16 & 1.76 & $\%$ \\
& Fasting glucose & 103.45 & 7.90 & $\mathrm{mg} / \mathrm{dl}$ \\
Lipid profile & Insulin & 6.01 & 2.65 & $\mathrm{units}$ \\
& Total Cholesterol & 167.80 & 35.69 & $\mathrm{mg} / \mathrm{dl}$ \\
& HDL-C & 57.60 & 13.46 & $\mathrm{mg} / \mathrm{dl}$ \\
Inflammatory profile & Triglyceride & 86.30 & 48.70 & $\mathrm{mg} / \mathrm{dl}$ \\
& LDL-C & 102.80 & 33.43 & $\mathrm{mg} / \mathrm{dl}$ \\
& VLDL-C & 7.40 & 5.40 & $\mathrm{mg} / \mathrm{dl}$ \\
& Leukocytes & 5.30 & 1.92 & $\mathrm{x} 10^{3} / \mu 1$ \\
& Neutrophil & 53.50 & 6.35 & $\times 10^{3} / \mu 1$ \\
& Eosinophil & 0.11 & 0.05 & $\times 10^{3} / \mu l$ \\
Hemogram & Lymphocyte & 34.10 & 6.38 & $\times 10^{3} / \mu \mathrm{l}$ \\
& im. Granulocytes & 0.35 & 0.21 & $\%$ \\
& hsCRP & 0.53 & 0.42 & $\mathrm{mg} / \mathrm{dl}$ \\
& Hemoglobin & 14.42 & 1.10 & $\mathrm{~g} / \mathrm{dl}$ \\
& Erythrocytes & 4.85 & 0.42 & $\mathrm{x} 10^{6} / \mu \mathrm{l}$ \\
& Hematocrit & 41.51 & 3.59 & $\%$ \\
& Platelets & 235.50 & 24.79 & $\mathrm{x} 10^{3} / \mu \mathrm{l}$ \\
\hline
\end{tabular}

The primary outcome measures were FMD (\%), measured at baseline, 2-, and 4-hours post-ingestion using AngioDefender (Everist Health, USA), as well as NO-dependent $\mathrm{NO}_{2} / \mathrm{NO}_{3}$ content in blood (using the Griess method), NOHb, lipid peroxidase, and CMA measures recorded at baseline, 1-, 2-, 3-, and 4-hours post-ingestion. The study was carried out according to the Helsinki declaration for clinical trials prior to 2008 and approved by Baden-Wuerttemberg Medical Association, Baden-Wuertemberg, Germany (F-2018-045) on $5^{\text {th }}$ of June 2018.

\subsection{Capsules}

The betalain-rich supplement BRS, a proprietary low nitrate food-based extract prepared from red beets was provided by VDF FutureCeuticals, Inc. (Momence, IL, USA). The phytochemical and nutritional composition of this patented low nitrate betalains-rich beet extract has been published previously (Nemzer et al., 2011). The composition of the active compounds in a single dose $(50 \mathrm{mg})$ of BRS is listed in Table 2. The organic pentaerythritol tetranitrate (PETN), the most commonly used nitrate for long-term treatment of patients exhibiting coronary artery disease was obtained from Altana Pharma $\mathrm{GmbH}$, Germany. PETN was included here as a positive control because of its standardized levels of nitrate and due to its known ability to increase NO. Red-colored gelatin capsules for the study were prepared by FutureCeuticals. Capsules were filled with micro cellulose (placebo), BRS or PETN. All capsule materials in the present study were of absolutely identical appearance and were stored in separately marked containers provided to the study subjects on the day of examination. 
Table 2. Phytochemical components of BRS

\begin{tabular}{lllll}
\hline Position & Phytochemical Components & Units & Amount (per 50 mg) & Amount (\%) \\
\hline 1 & Betalains & $\mathrm{mg}$ & 12.5 & 25 \\
2 & Nitrates & $\mathrm{mg}$ & 0.23 & 0.46 \\
3 & Sugars & $\mathrm{mg}$ & 0.15 & 0.30 \\
3 & Amino Acids & $\mu \mathrm{g}$ & 995 & 1.99 \\
& $\bullet \quad$ Arginine & $\mu \mathrm{g}$ & 193 & 0.39 \\
& $\bullet \quad$ Cystine & $\mu \mathrm{g}$ & 97.5 & 0.19 \\
4 & Vitamin C & $\mu \mathrm{g}$ & 307 & 0.62 \\
5 & Calcium & $\mu \mathrm{g}$ & 37.4 & 0.08 \\
6 & Iron & $\mu \mathrm{g}$ & 14.5 & 0.03 \\
\hline
\end{tabular}

\subsection{Primary Outcome Measures}

Flow-mediated dilation (FMD). The AngioDefender ${ }^{\circledR}$ device from Everist Health provides a new, non-invasive approach to assessing cardiovascular disease and aiding in heart disease prevention. By combining sensor technology with a software algorithm, and similar to the device provides gold standard brachial artery ultrasound imaging (BAUI) by measuring brachial artery vasodilator response (NO-dependent dilatation) as a surrogate prognostic predictor for endothelial function (Ras, Streppel, Draijer \& Zock, 2013; Flammer et al., 2012). Specifically, AngioDefender ${ }^{\circledR}$ performed analysis of the pulse wave before and after a 5-minute period of upper arm brachial artery occlusion. NO-dependent/flow-mediated vasodilation was calculated by applying the appropriate software algorithm related to the principles of plethysmography.

Bioavailable NO concentration assay $(\mathrm{NOHb})$. Blood was taken from a cubital vein using vacutainer containing L-Heparin without upper arm compression and was transferred into $1 \mathrm{ml}$ insulin syringe and spun down $(1600 \mathrm{x} \mathrm{g})$ for 5 mins at room temperature. Afterward, the sample was frozen in liquid nitrogen. Measurement of $\mathrm{NOHb}$ content was performed at $-196^{\circ} \mathrm{C}$ with liquid nitrogen-filled quartz finger dewar. ESR-spectrometer NOXYSCAN System equipped with a newly designed cavity operating at $86 \mathrm{kHz}$ field modulation was used to acquire ESR spectra at the $9.7 \mathrm{GHz} \mathrm{X}$ band with settings: microwave power, $40 \mathrm{~mW}$; modulation amplitude, 10 G; center field, $2.01 \mathrm{~g}$; sweep width, $240 \mathrm{G}$; conversion time, 320 milliseconds; time constant, 80 milliseconds; number of scans, 48; sweep time, 20.48 seconds (Dikalov \& Fink, 2005; Fink, Dikalov \& Fink, 2006). In short, quantification of $\mathrm{NOHb}$ was determined by comparison with a calibration curve generated by measuring the intensity of the EPR signal of erythrocytes that had been treated with known concentrations of nitrite $(1-25 \mu \mathrm{M})$. The amount of detected circulating $\mathrm{NOHb}$ concentration reflects the bioavailable level of $\mathrm{NO}^{\bullet}$ and, under physiological conditions, also represents the formation of $\mathrm{NO}^{\bullet}$ by eNOS (Dikalov \& Fink, 2005; Fink, Dikalov, \& Fink, 2006).

\subsection{Cellular Metabolic Activity Assay (CMA)}

Developed by Noxygen Science Transfer \& Diagnostics GmbH (Elzach, Germany), the "Cellular Metabolic Activity" (CMA) and its extension, "Extended CMA" assay, is based upon the monitoring of cellular, mitochondrial, peroxidase and NADPH-oxidase dependent generation of reactive oxygen species (ROS). Proper eNOS function and release of one $\mathrm{NO}^{\bullet}$ in a healthy endothelium requires L-arginine, 1.5 NADPH equivalents, and two oxygen molecules. As a consequence of excessive ROS formation, L-arginine causes "uncoupling" of eNOS wherein the oxygenase domain of eNOS releases superoxide radicals $\left(\mathrm{O} 2^{\bullet}\right)$ instead of $\mathrm{NO}^{\bullet}$ (Stroes, Hijmering, Zandvoort, Wever, Rabelink, \& Faassen, 1998). CMA assay was performed using bench-top EPR-spectrometer "NOXYSCAN System" equipped with a Temperature and Gas Controller (NOX-E.4-TGC, Noxygen Science Transfer \& Diagnostics GmbH, Germany) and a highly permeable spin probe (CMH, $200 \mu \mathrm{M})$. For measurement we added a spin probe with or without superoxide dismutase (SOD, $50 \mathrm{U} / \mathrm{ml}$ ), catalase (50 $\mathrm{U} / \mathrm{ml})$, or Antimycin A $(10 \mu \mathrm{M})$ to equal parts of $36 \mu \mathrm{L}$ freshly drawn capillary blood in order to perform all four types of analysis under controlled temperature and oxygen concentration $\left(\mathrm{t}=37^{\circ} \mathrm{C}, \mathrm{pO} 2=110 \mathrm{~mm} / \mathrm{Hg}\right)(\mathrm{Nemzer}$ et al., 2018). Parallel to the NOXYSCAN System, we performed the CMA analysis with the portable EPR analyzer VitaScan to verify the device for diagnostical and differential diagnostical purposes. Addition of an oxygen label (NOX-15.1 - $5 \mu \mathrm{M}$ ) to the blood sample allowed for monitoring of oxygen concentrations, in addition to cellular and mitochondrial oxygen consumption (Komarov, 2012). Here, we report NADPH-oxidase dependent generation of ROS (NOX- ROS) as well as cellular CMA (CMA). 


\section{$2.6 \mathrm{NO}_{2} / \mathrm{NO}_{3}$ using the Griess Assay}

The Griess assay is commonly used to detect NO, but it does not measure NO directly. We employed methods previously described by Shen et al., 2015. In short, we centrifuged the blood samples at $4{ }^{\circ} \mathrm{C}$, for 5 minutes at $600 \mathrm{~g}$ and employed $0.22 \mu \mathrm{M}$ filters in order to separate a small amount of plasma from the upper layer after centrifugation. Excess of $\mathrm{NO}^{\bullet}$ is rapidly converted into nitrite in the body by the interaction between $\mathrm{NO}^{\bullet}$ and $\mathrm{O}_{2} \cdot$. The Griess assay is run by a two-step diazotization reaction under acidic conditions in which the nitrite produces a diazonium ion. This ion is further processed to form a chromophoric azo derivative and monitored using spectrophotometric at $540 \mathrm{~nm}$ measurements. To calculate the level of nitrite in the plasma samples, we used the standard curve of a $\mathrm{NaNO}_{2}$ solution. The limitation of this method is that it does not measure $\mathrm{NO}^{\bullet}$ directly but measures accumulated oxidized $\mathrm{NO}^{\bullet}$ in the form of nitrite. Another limit of the assay is its rather

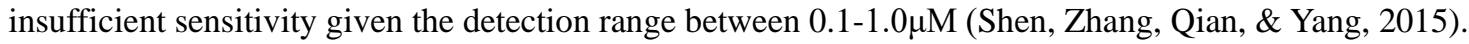

\subsection{Lipid-peroxide Detection}

Lipid-peroxide derivatives were measured in the same frozen blood pellet that was used for the detection of $\mathrm{NOHb}$ concentration using EPR-spectrometer NOXYSCAN at $-196^{\circ} \mathrm{C}$. In order to distinguish the lipid peroxide spectra from NOHb spectra, we acquired EPR spectra at two different microwave powers of 1 and 40mW. Delta of the signal between spectra acquired at $1 \mathrm{~mW}$ and oversaturated spectra at $40 \mathrm{~mW}$ was used for evaluation of oxygen-centered lipid-peroxide radical. The EPR spectrometer settings were as follows: microwave power, 1 or $40 \mathrm{~mW}$; modulation amplitude, 8G; center field, $2.03 \mathrm{~g}$; sweep width, $60 \mathrm{G}$; conversion time, $80 \mathrm{~ms}$; time constant, $20 \mathrm{~ms}$; the number of scans, 30 ; sweep time, $10.24 \mathrm{~s}$.

\subsection{Material and Chemicals}

The spin probes were 1-hydroxy-3-methoxycarbonyl-2.2.5.5-tetramethylpyrrolidine (CMH), 1-hydroxy-4-phosphono-oxy-2.2.6, 6-tetramethylpiperidine (PPH), the metal chelators deferoxamine (DF) and diethyldithiocarbamate (DETC). Krebs-Hepes buffer (KHB), and the oxygen label NOX-15.1 were obtained from Noxygen Science Transfer \& Diagnostics (Elzach, Germany). All other chemicals and reagents used were of analytical grade and were purchased from Sigma-Aldrich (St. Louis, MO. USA) unless otherwise specified.

\subsection{Statistical Analyses}

All outcome variables were tested for normality. Additionally, all variables were screened for extreme outliers, which were defined as $+/-2 *$ interquartile range (IQR) from the first or third quartiles. If outliers were identified, they were removed from the analysis. Data were checked for violations of assumptions for a repeated-measures ANOVA, including Mauchly's Test of Sphericity. In cases where sphericity was violated, Greenhouse-Geisser epsilon, the most conservative adjustment, was applied for correction of the degrees of freedom. For repeated measures ANOVAs, outcome variables at each timepoint were entered as within-subjects factors to determine the effect of time within each intervention. In the case of a significant omnibus repeated-measures ANOVA (i.e., $p<$ 0.05), post-hoc pairwise comparisons with Sidak multiple comparison correction were conducted. In outcome variables where normality was not achieved following removal of outliers, non-parametric Friedman Tests were conducted. All analyses were performed with SPSS Version 26 (IBM Corporation).

\section{Results}

\subsection{FMD}

A repeated measures ANOVA with FMD measurements following ingestion (i.e., prior to ingestion, 2 hours following ingestion, and 4 hours following ingestion) as a within-subjects factor demonstrated no significant changes for placebo $(F(2,18)=1.523, p=0.245)$ or PETN $(F(1.155,10.394)=2.615, p=0.134)$. There was a significant effect for $\operatorname{BRS}\left(F(2,18)=4.557, p=0.025\right.$, partial $\left.\eta^{2}=0.336\right)$. Pairwise comparisons with Sidak multiple comparison correction indicated that within 2 hours of ingestion, BRS significantly increased measures of FMD $(p=0.022)$ (Figure 1). There was no significant difference between the 2-hour and 4-hour post-ingestion measurements $(p=0.794)$, nor the baseline and 4-hour post-ingestion measures $(p=0.208)$. Of note, PETN had a significant test of sphericity (Mauchly's W $=0.268, p=0.005$ ), so Greenhouse-Geisser epsilon was applied for correction. These results suggest that under these experimental conditions only BRS significantly increased FMD within 2 hours of ingestion. 


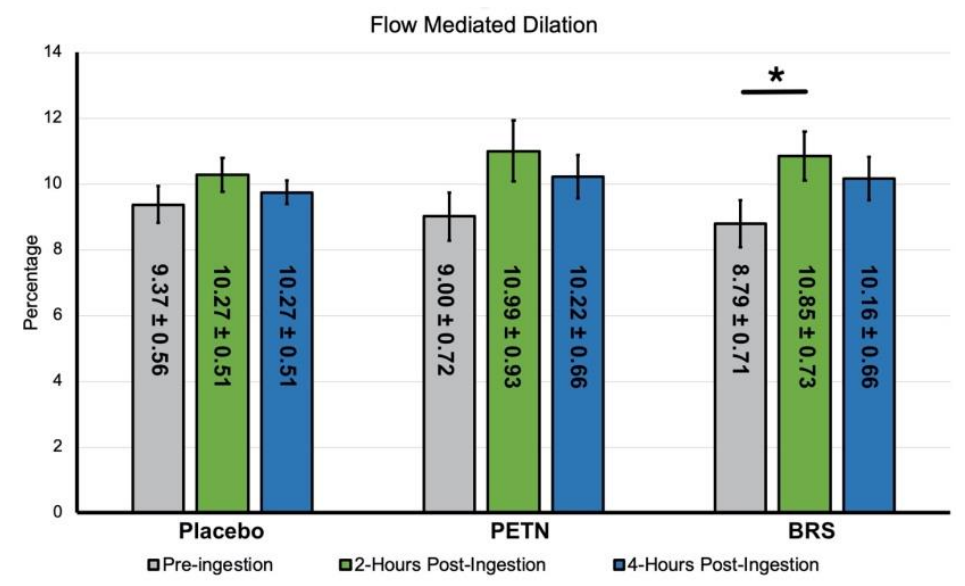

Figure 1. Time-dependent single-dose effect of BRS, placebo, and organic nitrate PETN on endothelial-dependent flow-mediated dilation (FMD). Green bars represent results 2-hours post-ingestions of Placebo, PETN, or BRS, while blue bars show results after 4-hours post-ingestion. Error bars represent SEM. * = $p<0.05$ in pairwise comparisons with Sidak multiple comparison correction following a significant omnibus repeated measures ANOVA. FMD is expressed as a percentage, with $M \pm S E M$ labels within the bars

$\mathrm{NOHb}$. Repeated measures ANOVAs were conducted with $\mathrm{NOHb}$ measurements following ingestion (i.e., prior to ingestion, 1-, 2-, 3-, and 4-hours following ingestion) as within-subjects factors demonstrated no significant changes in the Placebo condition $(F(1.491,13.416)=2.558, p=0.124)$ or the PETN condition $(F(4,36)=1.109$, $p=0.367)$. Of note, $\mathrm{NOHb}$ measurements consistently decreased over time in the Placebo condition, but for PETN, they trended towards an increase for 2 hours, after which they began to decrease. For BRS, however, there was a significant change in $\mathrm{NOHb}$ following ingestion $\left(F(2.246,20.210)=4.772, p=0.017\right.$, partial $\eta^{2}=$ 0.346). Pairwise comparisons with Sidak correction for multiple comparisons revealed that NOHb, after 2-hours, peaked, and was significantly different than after 4-hours $(p=0.001)$ (Figure 2). Of note, both Placebo and BRS conditions had significant tests of sphericity (Mauchly's $\mathrm{W}=0.045, p=0.007$; Mauchly's $\mathrm{W}=0.064, p=0.018$, respectively), so Greenhouse-Geisser epsilon corrections were applied. Together, these data suggest that under these experimental conditions only BRS demonstrated a significant effect of time on $\mathrm{NOHb}$ concentration. Specifically, increases peaked at 2-hours (32nM from baseline) and were significantly different between 2- and 4-hours post-ingestion.

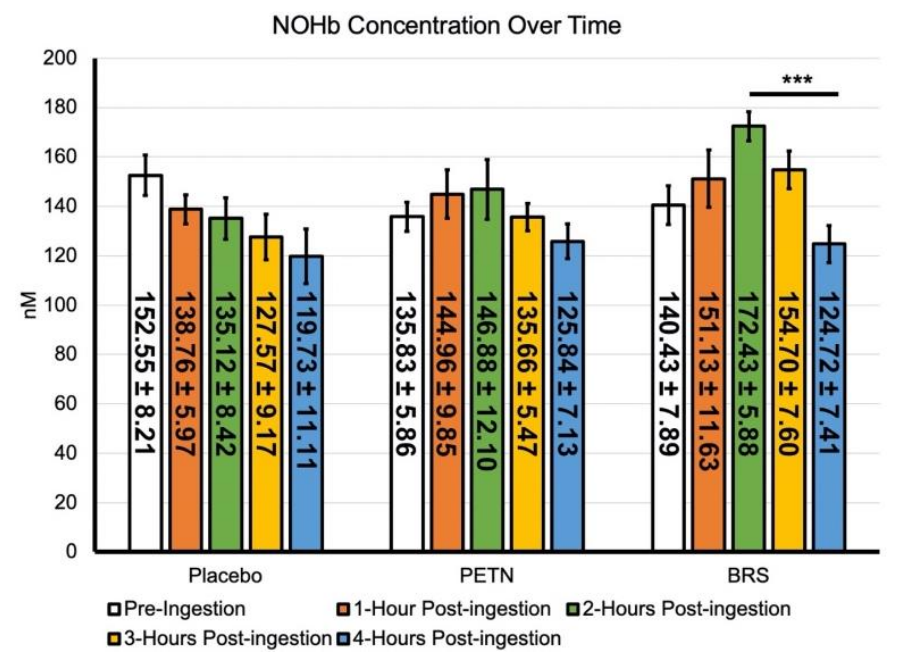

Figure 2. Time-dependent single dose effects of BRS, placebo, and organic nitrate PETN on circulating NOHb levels. Detection of circulating NOHb concentration was measured in venous blood using EPR-system NOXYSCAN System (for details, please see in the Methods section). Data are M $\pm S E M$. $* * *=p=0.001$ 


\subsection{NADPH-oxidase Dependent CMA (NOX-CMA)}

One outlier was identified within the NOX-CMA data in the PETN condition at the 1-hour post-ingestion mark. The outlier was removed from analyses. Assumptions were retested and both PETN and BRS had violations of normality, so we proceeded with a nonparametric Friedman Test. A repeated measures ANOVA revealed a significant effect for the placebo condition $\left(F(4,36)=3.982, p=0.009\right.$, partial $\left.\eta^{2}=0.307\right)$, but no significant effects were demonstrated for the PETN (Friedman Test: $X^{2}=1.133, N=9, p=0.889$ ) or BRS (Friedman Test: $X^{2}=3.358, N=10, p=0.500$ ) conditions. Pairwise comparisons with Sidak correction for multiple comparisons indicated that 4 hours post-ingestion was significantly different from pre-ingestion for the placebo condition $(\mathrm{p}=$ 0.003). Together these data suggest that under these experimental conditions neither BRS nor PETN significantly changed NADPH-dependent CMA but at two-hours post-ingestion BRS trended towards significance $(p=0.053)$. Please see Table 3 for descriptives.

Table 3. Descriptive statistics of time-dependent single-dose effect of BRS, placebo, and organic nitrate PETN on NADPH-dependent ROS generation

\begin{tabular}{llllllllllll}
\hline NADPH-dependent ROS Generation (nM/sec) \\
\hline & Pre-Ingestion & \multicolumn{1}{l}{ Pre-Ingestion } \\
\cline { 2 - 11 } & \multicolumn{1}{c}{} & & 1-Hours & 2-Hours & 3-Hours & 4-Hours \\
\cline { 2 - 11 } & $M$ & SEM & $M$ & SEM & $M$ & SEM & $M$ & SEM & $M$ & SEM \\
\hline Placebo & $-1.30^{* *}$ & 1.80 & 5.90 & 1.22 & 6.10 & 2.30 & 6.30 & 2.01 & 4.20 & 1.62 \\
PETN & -0.33 & 3.89 & 5.11 & 1.48 & 4.44 & 1.61 & 4.67 & 1.96 & 3.33 & 2.45 \\
BRS & -0.40 & 2.93 & 4.40 & 3.24 & 1.80 & 1.92 & 2.90 & 2.91 & 3.70 & 2.69 \\
\hline
\end{tabular}

$* *=$ difference between values at the level of $p<0.01$.

\subsection{CMA}

A repeated measures ANOVA with cellular CMA measurements following ingestion (i.e., prior to ingestion, 1-, 2-, 3-, and 4-hours following ingestion) as within-subjects factors demonstrated significant changes for the placebo condition $\left(F(4,36)=5.091, p=0.002\right.$, partial $\left.\eta^{2}=0.361\right)$, with pairwise comparisons with Sidak multiple comparison correction indicating that 1-hour post-ingestion of placebo was significantly different than 4-hours post-ingestion $(p=0.008)$. Specifically, placebo CMA significantly decreased between the 1-hour post-ingestion measurement and the 4-hour post-ingestion measurement. Under these experimental conditions no significant changes were noted for the $\operatorname{PETN}(F(4,36)=0.708, p=0.592)$ or $\operatorname{BRS}(F(4,36)=2.377, p=0.070)$ groups. Descriptive statistics are presented in Table 4.

Table 4. Descriptive statistics of time-dependent single dose effect of betalain-rich AltRed ${ }^{\mathrm{TM}}$ and organic nitrate PETN on total CMA

\begin{tabular}{llllllllllll}
\hline Cellular Metabolic Activity (nM/sec) \\
\hline & Pre-Ingestion & \multicolumn{1}{l}{ Post-Ingestion } \\
\cline { 2 - 13 } & & & 1-Hours & 2-Hours & 3-Hours & 4-Hours & \\
\cline { 2 - 13 } & $M$ & SEM & $M$ & SEM & $M$ & SEM & $M$ & SEM & $M$ & SEM \\
\hline Placebo & 230.20 & 2.07 & $231.40^{* *}$ & 2.94 & 225.70 & 2.41 & 228.30 & 2.83 & $224.40^{* *}$ & 2.00 \\
PETN & 232.60 & 5.21 & 229.90 & 2.89 & 226.00 & 3.52 & 228.40 & 3.15 & 226.50 & 3.02 \\
BRS & 233.20 & 3.77 & 230.40 & 3.63 & 227.60 & 2.71 & 224.30 & 2.19 & 226.30 & 2.36 \\
\hline
\end{tabular}

\section{$3.4 \mathrm{NO}_{2} / \mathrm{NO}_{3}$}

Two outliers were identified within the $\mathrm{NO}_{2} / \mathrm{NO}_{3}$ data. One outlier was removed from the placebo data and one from the BRS data, both from the 4-hour post-ingestion measures. A repeated measures ANOVA for $\mathrm{NO}_{2} / \mathrm{NO}_{3}$ measurements within each condition revealed a significant effect of time for all conditions (Placebo: $F(4,36)=$ 4.233, $p=0.007$, partial $\eta^{2}=0.320$; PETN: $F(2.292,18.338)=3.447, p=0.048$, partial $\eta^{2}=0.301$; BRS: $F(4,32)$ $=4.951, p=0.003$, partial $\eta^{2}=0.382$ ) (Figure 3). Pairwise comparisons with Sidak multiple comparison correction did not reveal differences for the placebo or BRS conditions but did reveal a significant difference between baseline and 2-hours post-ingestion for PETN $(p=0.049)$. The difference between baseline and 3-hours post-ingestion approached significance for the BRS condition $(p=0.061)$. Of note, PETN had a significant test of sphericity (Mauchly's W $=0.043, p=0.020$ ), so Greenhouse-Geisser epsilon corrections are reported. Together these data suggest that under these experimental conditions only PETN significantly increased 
$\mathrm{NO}_{2} / \mathrm{NO}_{3}$ at 2 hours although BRS trended towards significance at three hours.

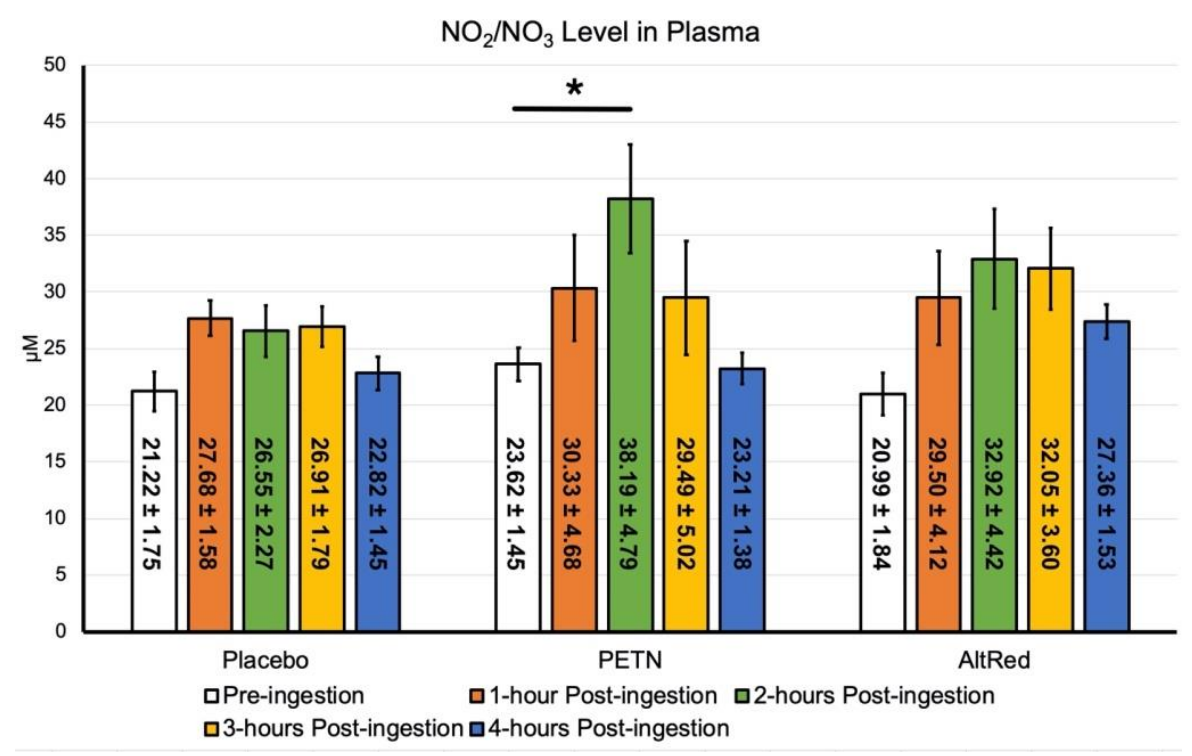

Figure 3. Time-dependent single dose effect of BRS, placebo, and PETN on NO2/NO3 content in blood plasma

White, orange, green, yellow, and blue bars show results pre-ingestion, 1-hour, 2-hours, 3-hours, and 4-hours post-ingestion. Data are $M \pm S E M . *=p<0.05$.

\subsection{Lipid Peroxide}

A repeated measures ANOVA with lipid peroxide concentration measurements following ingestion (i.e., prior to ingestion, 1-, 2-, 3-, and 4-hours following ingestion) as within-subjects factors revealed that under these experimental conditions there were no significant effects in any of the conditions (Placebo: $F(4,36)=0.944, p=$ 0.450 ; PETN: $F(2.059,18.529)=2.839, p=0.083$; BRS: $(F(4,36)=0.375, p=0.825)$. Of note, the PETN condition data had a significant test of sphericity (Mauchly's $\mathrm{W}=0.085, p=0.036$ ), so Greenhouse-Geisser epsilon corrections were applied.

Table 5. Descriptive statistics for time-dependent single dose effects of BRS, placebo, and organic nitrate PETN on cell membrane lipid peroxidation

\begin{tabular}{|c|c|c|c|c|c|c|c|c|c|c|}
\hline \multicolumn{11}{|c|}{ Lipid Peroxide Concentration $(\mu \mathrm{m})$} \\
\hline & \multirow{2}{*}{\multicolumn{2}{|c|}{ Pre-Ingestion }} & \multicolumn{8}{|c|}{ Post-Ingestion } \\
\hline & & & \multicolumn{2}{|c|}{ 1-Hours } & \multicolumn{2}{|c|}{ 2-Hours } & \multicolumn{2}{|c|}{ 3-Hours } & \multicolumn{2}{|c|}{ 4-Hours } \\
\hline & $M$ & SEM & $M$ & SEM & $M$ & SEM & $M$ & SEM & $M$ & SEM \\
\hline Placebo & 10.58 & 1.51 & 14.66 & 2.17 & 13.12 & 2.07 & 15.69 & 2.82 & 13.07 & 2.53 \\
\hline PETN & 9.99 & 1.35 & 13.99 & 3.36 & 16.90 & 3.21 & 12.63 & 2.92 & 9.56 & 1.00 \\
\hline BRS & 12.10 & 2.20 & 14.08 & 2.91 & 15.91 & 4.66 & 15.38 & 1.58 & 14.88 & 3.49 \\
\hline
\end{tabular}

Data are $M \pm S E M$.

\section{Discussion}

Interest in products to increase nitric oxide production and blood flow continues to grow. Many athletes are engaged in ingesting plant-based materials that are high in dietary nitrates in hopes of improving performance. However, published manuscripts describing contraindicative results of long-term ingestion of high levels of nitrates. Consequently, alternative, low nitrate plant-based materials could be beneficial for individuals seeking to increase NO levels and blood flow. Nitroglycerine and other organic nitrates have been used therapeutically as vasodilators for the treatment of "angina pectoris" to support the impaired generation of nitric oxide associated with dysfunctional endothelium. However, pharmaceutical modalities cannot be used for athletic enhancement or for everyday support for endothelial health. In this clinical trial, we compared the effects of a nutraceutical, betalains-enriched low nitrate supplement, BRS, against a high nitrate active control (PETN) and placebo on parameters of endothelial function at the cellular level. Specifically, we tested primary outcomes related to 
cardiovascular-relevant measures such as $\mathrm{FMD}, \mathrm{NOHb}, \mathrm{NO}_{2} / \mathrm{NO}_{3}, \mathrm{CMA}$, and lipid peroxide concentrations.

As hypothesized, these data show a statistically significant, time-dependent elevation of circulating $\mathrm{NOHb}$ concentrations and endothelial-dependent FMD after 2 hours of BRS administration. The NOHb results are in alignment with previous data demonstrating $\mathrm{t}_{1 / 2}$ of 3 hours for the bioconversion of PETN to PETriN (Schütz, Kötting, Epple, Ziegler, Maier-Lenz, \& Schütz, 1999). Contrary to our expectations, we did not find a similar significant NOHb increase in the PETN group, although PETN trended towards significance at 2 hours. These data suggest that a low nitrate, betalain-rich material (BRS) may outperform a known high nitrate material (in this case PETN) with regard to elevation of levels of bioavailable nitric oxide (i.e., NOHb). In the placebo group, and in contrast to the administration of PETN or BRS we observed a continuous qualitative decrease of circulating $\mathrm{NOHb}$ concentration, providing preliminary support that PETN and BRS are acting over the elevation of bioavailable $\mathrm{NO}^{\bullet}$.

Measured increases in $\mathrm{NO}_{2} / \mathrm{NO}_{3}$ are often used as an indirect proxy for possible evidence of increases in nitric oxide levels pre-breakdown to $\mathrm{NO}_{2} / \mathrm{NO}_{3}$ in blood plasma. As hypothesized, only the PETN condition had statistically significant differences on $\mathrm{NO}_{2} / \mathrm{NO}_{3}$ between pre-ingestion and 2-hours post-ingestion. Specifically, ingestion of PETN resulted in a statistically significant increase in $\mathrm{NO}_{2} / \mathrm{NO}_{3}$ after 2 hours, and this correlated (although only as a trend) with PETN's 2-hour time point of maximal liberation of nitric oxide measured as circulating $\mathrm{NOHb}$ level (Figure 2), presumably due to the prevalence of extracellular bioconversion of this organic nitrate. Interestingly, while the observed results show that a single-dose of BRS did not statistically increase $\mathrm{NO}_{2} / \mathrm{NO}_{3}$ level in plasma (Figure 3), BRS did show a statistically significant increase in $\mathrm{NOHb}$. These observed increases of endogenous NOHb coupled with resulting improvements in FMD using an essentially nitrate-free BRS were observed here for the first time, and these data suggest the low nitrate betalains may naturally regulate $\mathrm{NO}-$ dependent vasomotion.

Finally, we examined eNOS function and ROS generation. Contrary to our hypotheses, only the placebo group demonstrated any significant change wherein we observed an elevation of NADPH-dependent ROS and total CMA. One potential reason for this elevated NADPH-oxidase activity, which is regulated by laminar blood flow, may be a consequence of the physical manipulation and implementation of the FMD measurement that by definition required 10 min of no-flow ischemia. Such ischemia induces NADPH-oxidase dependent generation of $\mathrm{O}_{2} \bullet$, that at the same time were at least somewhat counteracted by BRS- and PETN-induced NO ${ }^{\bullet}$ formation.

Previous investigations reported that dietary redox-active/antioxidant betalains indicaxanthine act through inhibition of NADPH oxidase and intracellular signaling modulation leading to the inactivation of NF-kB, as well as modulation of inducible nitric oxide synthase expression (Tesoriere, Attanzio, Allegra, Gentile, \& Livrea, 2014). Although our hypotheses for BRS and PETN related to NADPH-dependent ROS, total CMA and lipid peroxidase were incorrect under these experimental conditions, our findings somewhat confirmed the inhibitory capacity of betalains on NADPH oxidase. However, this effect alone, represented by a reduction of ROS generation up to $5 \mathrm{nM} / \mathrm{sec}$, is not sufficient to induce an increase of circulating NOHb levels up to $46.2 \pm 12 \mathrm{nM}$ after 2 hours following BRS administration. Consequently, this confirms our assumption of additional unidentified regulatory mechanisms induced by betalains related to modulation of eNOS activity.

This pilot study has some limitations. First, the sample size is small and thus the study has limited power. As such, larger samples could help to more robustly characterize the effects of BRS on other measures of endothelial function, especially as relates to ROS. In balance, however, the crossover aspect of the design helped to improve the significance of the limited sample size. Future studies are planned that will include a larger sample size and an increased age range for greater generalizability. Second, we did not record dietary changes between days, nor were diets monitored. However, the fasting window likely mitigates any potential influence that dietary differences would have between conditions. Third, it is important to point out that FMD follows diurnal rhythm changes, so these may have had some impact on our results (Jones, Lewis, Thompson, Marrin, Green, \& Atkinson, 2012). However, all conditions were performed under the same timeframes, and thus, this effect should hopefully be somewhat blunted by the nature of our study design. Fourth, it may well be that FMD, due to previously noted ischemic challenges, may not be the best testing criteria partner to include when conducting real-time ROS/CMA testing. Fifth, a narrowing of some of the intake criteria may yield further insights. Finally, additional, well-powered studies can be conducted to replicate and hopefully expand the results presented here, and to better understand the physiological mechanisms subserving the potential health benefits of a high betalain, low nitrate, low sugar material, BRS.

\section{Conclusion}

Here, we provide preliminary data from a double-blinded, randomized, crossover, placebo-controlled 
comparative clinical trial assessing the effects of betalains-rich low nitrate supplement (BRS) in comparison to organic nitrate PETN. The results show the potency of BRS to increase the blood level of bioavailable nitric oxide as $\mathrm{NOHb}$ concentration. Consequently, this also increased the endothelium-dependent flow-mediated vasodilation (FMD) in a manner superior to therapeutically used, slow-release organic nitrate PETN without side effects such as induction of lipid peroxidation and nitrite/nitrate formation. These effects may naturally contribute to increased sports performance and increased power output stimulated by BRS as reported in previous clinical studies; however, these data need to be replicated and expanded. The findings justify further need for clinical investigations to explore the potential beneficial effects of BRS on conditions requiring improved vascular endothelial functions and $\mathrm{NO}^{\bullet}$ dependent processes such as $\mathrm{O}_{2}$ uptake, blood circulation, and blood distribution. These early data suggest an exciting possible alternative to conventional wisdom, current understanding and commonly ingested high-nitrate products. They certainly suggest that the chemistry of betalains may provide new and deeper insights into sports and cardiovascular nutrition.

\section{Conflicts of interest and disclosures}

\section{Funding}

This study, project \#F-2018-045 was financially supported by VDF FutureCeuticals, Inc. Noxygen GmbH, the study provider, was contracted to conduct the protocol.

\section{Author contributions}

BF designed the study protocol, performed clinical examinations, and edit the manuscript. BVN, ZP and JMH assisted in the design of the study protocol and contributed to the study write-up. JLR performed independent data analyses and contributed to the study write-up and editing.

\section{Conflict of interest}

BVN, ZP and JMH are employees of VDF FutureCeuticals, Inc. BF is an employee of Noxygen, JLR is an independent consultant who was compensated by VDF FutureCeuticals, Inc. This study was double-blinded in order to minimize all potential conflicts.

\section{Acknowledgments}

We are grateful to Patricia Dufner for her excellent technical support.

\section{References}

Bassenge, E., Fink, N., Skatchkov, M., \& Fink, B. (1998). Dietary supplement with vitamin C prevents nitrate tolerance. The Journal of Clinical Investigation, 102, 67-71. https://doi.org/10.1172/JCI977

Beckman, J. S., \& Koppenol, W. H. (1996). Nitric oxide, superoxide, and peroxynitrite: the good, the bad, and ugly. American Journal of Physiology-Cell Physiology, 271, C1424-C1437. https://doi.org/10.1152/ajpcell.1996.271.5.C1424

Bibli, S. I., Hu, J., Leisegang, M. S., Wittig, J., Zukunft, S., Kapasakalidi, A., ... Fleming, I. (2020). Shear stress regulates cystathionine $\gamma$ lyase expression to preserve endothelial redox balance and reduce membrane lipid peroxidation. Regulation of CSE by KLF2 and miR-27b. Redox Biology, 28, 101379. https://doi.org/10.1016/j.redox.2019.101379

Dikalov, S., Fink, B., Skatchkov, M., \& Bassenge, E. (1999). Comparison of glyceryl trinitrate-induced with pentaerythrityl tetranitrate-induced in vivo formation of superoxide radicals: effect of vitamin C. Free Radical Biology and Medicine, 27, 170-176. https://doi.org/10.1016/S0891-5849(99)00066-0

Dikalov, S., \& Fink, B. (2005). ESR Techniques for the Detection of Nitric Oxide In Vivo and in Tissues. Methods in Enzymology, 396, 597-610. https://doi.org/10.1016/S0076-6879(05)96052-7

Doehner, W., \& Landmesser, U. (2011). Xanthine oxidase and uric acid in cardiovascular disease: Clinical impact and therapeutic options. Seminars in Nephrology, 31(5), 433-440. https://doi.org/10.1016/j.semnephrol.2011.08.007

Doughan, A. K., Harrison, D. G., \& Dikalov, S. I. (2008). Molecular Mechanisms of Angiotensin II-Mediated Mitochondrial Dysfunction. linking mitochondrial oxidative damage and vascular endothelial dysfunction. Circulation Research, 102, 488-496. https://doi.org 10.1161/CIRCRESAHA.107.162800

Fink, B., \& Bassenge, E. (1997). Unexpected, tolerance-devoid vasomotor and platelet actions of pentaerythrityl tetranitrate. Journal of Cardiovascular Pharmacology, 30, 831-836. https://doi.org/10.1097/00005344-199712000-00020 
Fink, B., \& Bassenge, E. (2002). Association between vascular tolerance and platelet upregulation: comparison of nonintermittent administration of pentaerithrityl tetranitrate and glyceryltrinitrate. Journal of Cardiovascular Pharmacology, 40, 890-897. https://doi.org/10.1097/00005344-200212000-00010

Fink, B., Dikalov S., \& Fink, N. (2006). ESR techniques for the detection of nitric oxide in vivo as an index of endothelial function. Pharmacological Reports, 58, 8-15.

Flammer, A. J., Anderson, T., Celermajer, D. S., Creager, M. A., Deanfield, J., Ganz, P., ... Hamburg, N. M. (2012). The assessment of endothelial function: from research into clinical practice. Circulation, 126, 753-767. https://doi.org/10.1161/CIRCULATIONAHA.112.093245

Griendling, K. K., Sorescu, D., \& Ushio-Fukai, M. (2000). NAD(P)H Oxidase. Role in Cardiovascular Biology and Disease. Circulation Research, 86, 494-501, https://doi.org/10.1161/01.RES.86.5.494

Hooreebeke, J., Trias, C., Davis, B., Lozada, C., \& Casazza, G. (2016). Betalain-rich concentrate supplementation improves exercise performance in competitive runners. Sports, 4(40), 1-9. https://doi.org/10.3390/sports4030040

Hurtado-Barroso, S., Quifer-Rada, P., Fernando, J., de Alvarenga, R., Pérez-Fernández, S., Tresserra-Rimbau, A., \& Lamuela-Raventos, R. M. (2018). Changing to a low-polyphenol diet alters vascular biomarkers in healthy men after only two weeks. Nutrients, 10 . https://doi.org/10.3390/nu10111766

Jones, H., Lewis, N. C. S., Thompson, A., Marrin, K., Green, D., \& Atkinson, G. (2012). Diurnal variation in vascular function: Role of sleep. Chronobiology International, 29, 271-277. https://doi.org/10.3109/07420528.2012.654554

Lacy, F., O'Connor, D. T., \& Schmid-Schönbein, G. W. (1998). Plasma hydrogen peroxide production in hypertensives and normotensive subjects at genetic risk of hypertension. Journal of Hypertension, 16, 291-303. https://doi.org/10.1097/00004872-199816030-00006

Landmesser, U., Cai, H., Dikalov, S., McCann, L., Hwang, J., Jo, H., Holland, S. M., \& Harrison, D. G. (2002). Role of $\mathrm{p} 47^{\text {phox }}$ in vascular oxidative stress and hypertension caused by angiotensin II. Hypertension, 40, 511-515. https://doi.org/10.1161/01.HYP.0000032100.23772.98

Laude, K., Cai, H., Fink, B., Hoch, N., Weber, D. S., McCann, L., ... Harrison, D. G. (2005). Hemodynamic and biochemical adaptations to vascular smooth muscle overexpression of $\mathrm{p} 22^{\text {phox }}$ in mice. American Journal of Physiology-Heart and Circulatory Physiology, 288, H7-H12. https://doi.org/10.1152/ajpheart.00637.2004

Kanner, J., Harel, S., \& Granit, R. (2001). Betalains - A new class of dietary cationized antioxidants. Journal of Agricultural and Food Chemistry, 49, 5178-5185. https://doi.org/10.1021/jf010456f

Kleinbongard, P., Dejam, A., Lauer, T., Rassaf, T., Schindler, A., Picker, O., ... Kelm, M. (2003). Plasma nitrite reflects constitutive nitric oxide synthase activity in mammals. Free Radical Biology and Medicine, 35, 790-796. https://doi.org/10.1016/S0891-5849(03)00406-4

Kleinbongard, P., Dejam, A., Lauer, T., Jax, T., Kerber, S., Gharini, P., ... Kelm, M. (2006). Plasma nitrite concentrations reflect the degree of endothelial dysfunction in humans. Free Radical Biology and Medicine, 40, 295-302. https://doi.org/10.1016/j.freeradbiomed.2005.08.025

Komarov, D. A., Dhimitruka, I., Kirilyuk, I. A., Trofimiov, D. G., Grigor'ev, I. A., Zweier, J. L., \& Khramtsov, V. V. (2012). Electron paramagnetic resonance monitoring of ischemia-induced myocardial oxygen depletion and acidosis in isolated rat hearts using soluble paramagnetic probes. Magnatic Resonance in Medicine, 68, 649-655. https://doi.org/10.1002/mrm.23251

McDonald, J. D., Chitchumroonchokchai, C., Li, J., Mah, E., Labyk, A. N., Reverri, E. J., Ballard, K. D., Volek, J. S., \& Richard, S. (2018). Replacing carbohydrate during a glucose challenge with the egg white portion or whole eggs protects against postprandial impairments in vascular endothelial function in prediabetic men by limiting increases in glycaemia and lipid peroxidation. British Journal of Nutrition, 119, 259-270. https://doi.org/10.1017/S0007114517003610

Mendonça, R. D., Carvalho, N. C., Martin-Moreno, J. M., Pimenta, A. M., Lopes, A. C. S., Gea, A., ... Bes-RastrolloMendonça, M. (2018). Total polyphenol intake, polyphenol subtypes and incidence of cardiovascular disease: The SUN cohort study. Nutrition, Metabolism and Cardiovascular Diseases, 29, 69-78. https://doi.org/10.1016/j.numecd.2018.09.012

Montenegro, C., Kwong, D., Minow, Z., Davis, B., Lozada, C., \& Casazza, G. (2017). Betalain-rich concentrate supplementation improves exercise performance and recovery in competitive triathletes. Applied Physiology, 
Nutrition, and Metabolism, 42, 166-172. https://doi.org/10.1139/apnm-2016-0452

Nemzer, B. V., Pietrzkowski, Z., Spórna, A., Stalica, P., Thresher, W., Michałowski,T., \& Wybranie, S. (2011). Betalainic and nutritional profiles of pigment-enriched red beet root (Beta vulgaris L.) dried extracts. Food Chemistry, 127, 42-53. https://doi.org/10.1016/j.foodchem.2010.12.081

Nemzer, B. V., Fink, N., \& Fink, B. (2014). New insights on effects of a dietary supplement on oxidative and nitrosative stress in humans. Food Science and Nutrition, 2(6), 828-839 https://doi.org/10.1002/fsn3.178

Nemzer, B. V., Centner, C., Zdzieblik, D., Fink, B., Hunter, J. M., \& König, D. (2018). Oxidative stress or redox signalling - new insights into the effects of a proprietary multifunctional botanical dietary supplement. Free Radical Research, 52, 362-372. https://doi.org/10.1080/10715762.2017.1390228

Ras, R. T., Streppel, M. T., Draijer, R., \& Zock, P. L. (2013). Flow-mediated dilation and cardiovascular risk prediction: A systematic review with meta-analysis. International Journal of Cardiology, 168, 344-351. https://doi.org/10.1016/j.ijcard.2012.09.047

Ramana, K. V., Srivastava, S., \& Singhal, S. S. (2014). Lipid peroxidation products in human health and disease 2014. Oxidative Medicine and Cellular Longevity, 162414-162414. https://doi.org/10.1155/2014/162414

Schütz, A., Kötting, J., Epple, F., Ziegler, R., Maier-Lenz, H., \& Schütz, D. (1999). Quantitative gaschromatographische / massenspektrometrische bestimmung der pentaerithrityltetranitrat-metaboliten pentaerithrityltrinitrat, pentaerithrityldinitrat und pentaerithritylmononitrat in humanplasma. Arzneimittelforschung, 49, 891-895. https://doi.org/10.1055/s-0031-1300522

Shen, Y., Zhang, Q., Qian, X., \& Yang, Y. (2015). Practical assay for nitrite and nitrosothiol as an alternative to the Griess assay or the 2,3-Diaminonaphthalene assay. Analytical Chemistry, 87, 1274-1280. https://doi.org/10.1021/ac5039779

Stroes, E., Hijmering, M., Zandvoort, M. V., Wever, R., Rabelink, T. J., \& Faassen, E. E. V. (1998). Origin of superoxide production by endothelial nitric oxide synthase. FEBS Letters, 438, 161-164. https://doi.org/10.1016/S0014-5793(98)01292-7

Tesoriere, L., Attanzio, A., Allegra, M., Gentile, C., \& Livrea, M. A. (2014). Indicaxanthin inhibits NADPH oxidase (NOX)-1 activation and NF- $\kappa B-d e p e n d e n t$ release of inflammatory mediators and prevents the increase of epithelial permeability in IL-1 $\beta$-exposed Caco-2 cells. British Journal of Nutrition, 111, 415-423. https://doi.org/10.1017/S0007114513002663

\section{Copyrights}

Copyright for this article is retained by the author(s), with first publication rights granted to the journal.

This is an open-access article distributed under the terms and conditions of the Creative Commons Attribution license (http://creativecommons.org/licenses/by/4.0/). 